\title{
Disassembly Planning Based on Precedence Relations among Assemblies
}

\author{
F. Torres ${ }^{1}$, S. T. Puente ${ }^{1}$ and R. Aracil ${ }^{2}$ \\ ${ }^{1}$ Department of Physics, Systems Engineering and Signal Theory, University of Alicante, Spain; ${ }^{2}$ DISAM, Polytechnic University of \\ Madrid, Spain
}

\begin{abstract}
An algorithm is presented that is based on a representation method for products, which lets us show, intuitively, the hierarchical relationships among components and/or assemblies of the product. This paper presents an algorithm for establishing a partial non-destructive disassembly sequence of a product. The disassembly sequence can be obtained for a component as well as for a sub-assembly (group of different components). The disassembly sequence is obtained autonomously and automatically and can be used in a flexible manufacturing system.
\end{abstract}

Keywords: Disassembly planning; Flexible manufacturing

\section{Introduction}

The key to automatic recycling processes or to industrial maintenance is non-destructive disassembly. The establishment of the correct operations sequence is the step previous to achieving an automatic non-destructive disassembly of a product, so that only the partial disassembly of the product is done, and with just the operations that are strictly necessary; for example, if we want to change or remove the battery in a car, the only operations that are required are the ones that give us access to the battery.

We need to know the components that form the product and the relationships among them, to be able to determine a disassembly sequence for the product. If a disassembly for the recycling of the product's components is to be done, then it is necessary to reflect the different materials of the components in the representation, because the joint disassembly of two components is only correct if they are both made of the same material. If a disassembly for maintenance is to be done, then a representation that reflects the different components to be changed and their relationships, instead of their different materials, would be better.

Correspondence and offprint requests to: F. Torres, Department of Physics, Systems Engineering and Signal Theory, University of Alicante, Crta San Vicente s/n, PO Box 99, Alicante, Spain. E-mail: ftorres@disc.ua.es
In this paper, we present a representation based on assemblies of components, grouped by hierarchical levels, that form an assembly. These can be considered as new components in other groups or assemblies. This type of representation, in addition to being intuitive, affords us a connection among operations or tasks to be done for the real disassembly, by automatically generating the sequence of operations to achieve the disassembly of a component or of a sub-assembly of the product.

The following section of the paper presents a state-of-theart product representation for disassembly. Afterwards, the different concepts are defined. The representation and method for the disassembly are then presented. The experimental results are presented and, finally, the conclusions are given.

\section{Product Representation}

This paper presents an algorithm that automatically establishes an optimal partial disassembly sequence for a product, using non-destructive techniques derived from a new intuitive product representation.

There are different schemes for presenting a product, depending on what we want to reflect. There are schemes that try to present the product from a designer's point of view, from a user's point of view, from relationships among different components or from components characteristics, etc. Therefore, to select a type of product representation for disassembly, a study has to be made of the product's characteristics to be reflected in the scheme, the type of disassembly to be achieved, who has to understand the representation, and so on.

The characteristics of the product to be represented have an influence on the representation; i.e., it changes the granularity of the components that make up the product. As an example, in a PC, cards and hard disks can be taken into consideration as components but not individual chips, which form the cards or hard disks. Chips cannot be disassembled with this representation, but if this is not desired the disassembly level of the scheme is valid and simpler than taking these characteristics into consideration. 
Several types of product representations are described, as follows [1]:

- Using a labelled graph with four different types of labels (parts, contacts, attachments and relations) in which the solid pieces of the products are the components. Contacts represent the link between components, without external elements. Attachments represent the physical pieces that make contact. Relations represent associations among parts, contacts and attachments [2]. This type of representation is useful if all the components are perfectly well known as well as the relations among them. On the other hand, the representation is too complicated to be done in a simple way.

- Using a hierarchical representation of the product by a graph in which nodes represent links between the surfaces of the components. Each node has a label which indicates the disassembly direction for removing that node. If it is a terminal node, it can be disassembled directly. Edges represent precedence relationships among components. As the components are disassembled, and the graph is simplified, the nodes change their label to express this change [3] . This method implies, like the previous one, a detailed knowledge of the product as well as of the different possibilities of movement for each component, relative to the others.

- Using the geometric characteristics of the products [4], the problem can be resolved by several methods since, with this information, geometric, topological and technological restrictions can be inferred. With this information, the user iteration is reduced and the information needed by the system to generate the disassembly sequences is also reduced [5-7]. The position information must be exact for each component as well as its relation to the rest of the components. For this type of method a precise geometric model of every component is needed.

One approach is based on generating a disassembly graph, using the geometrical information which when followed achieves the disassembly sequence.

Another approach consists of obtaining an iterative solution by wave propagation [8-10]. Two disadvantages of these methods are that they only achieve good results for 1disassembly. When a component needs $n$ independent movements to be disassembled, it is an n-disassembly systems. When they change from two-dimensional systems to three-dimensional ones the complexity in computing a solution increases enormously.

- Another approach is based on generating a tree with each possible disassembly sequence [11]. This method uses the weight assignment to each branch of the tree for choosing the strategy for disassembling the product in real time. If the algorithm arrives at a branch where it can keep on disassembling other trees that were previously generated, one is chosen and it keeps on disassembling, based on this new information. This strategy is designed for application in real-time systems. One disadvantage is that if the product is complex the strategy will be impossible to use, since a tree has to be saved for each possible disassembly sequence.

- The use of and/or graphs is a compact representation of the product as well as the relationships among components [12-
15]. The nodes of the graph are groups of components and the leaves correspond to individual components. By merging two leaves, an intermediate node is achieved which represents a sub-assembly of the product. By combining these new sub-assembly nodes with the rest of the graph's nodes, nodes with more components are obtained. This process continues until it arrives to the root node, which represents the completely assembled product. The disassembly sequence is generated from the root to the component that is to be disassembled. This method has the advantage of being an intuitive representation of the product. However, in complex products the graph can be large and difficult to represent.

- Using a graph made with geometric, topological and functional data of the product, which represents relations among contacts and components of the product [16]. This type of representation has a lot of product information, so that it is easy to obtain a disassembly sequence when the graph is generated. The need for a lot of information to generate the graph implies a lot of user iteration to obtain the information.

- Using a components-joins graph, whose nodes correspond to the components, and whose edges represent joins between components [17-19]. This method derives the disassembly sequence after transforming the graph into a tree, which represents an order in which the different components are to disassembled, since the problem is reduced to one search within one tree. The principal advantage of this method is that it simplifies searching in a tree instead of within a graph, thus reducing the search time. Neither the product graph representation nor its relations are intuitive, and it needs a great knowledge of the product to be able to represent it accurately.

- Another type of product representation is based on the definition of relations among groups of components using clusters [20]. The product graph distributes the components into layers, where it is needed to disassemble one layer before disassembling any of the components of the next layer. The graph edges represent precedence relations between the components in different layers. When the layers and the relations are defined, the components are grouped into clusters, taking the different sub-assemblies of the product into consideration. This representation has the advantage of limiting the relations among components of the product. On the other hand, the need to correctly sort the components by layer requires a wide knowledge of the product.

- Using direct graphs whose nodes are components, and whose edges are relationships between components [21]. This representation takes three different types of relationship between components into consideration. It has the advantages of being an easy representation for an operator, and it uses a cost function defined analytically for computing the disassembly sequence. It requires a great knowledge of the relations between the components to be able to define the product graph.

This paper presents another approach for representing a product, based on the relations among components that define new components (assemblies), since a product can be expressed as a union of sets of assemblies with hierarchical 
relations among them. This method has the advantage over the previous ones of being an easy, intuitive representation of the product. In addition, it permits an increase of the granularity level of a representation, based on a previous one. It uses a set of rules for obtaining the disassembly sequence of the different types of assemblies, depending on which component is to be disassembled. The disassembly problem is then reduced to finding which assemblies have the component to be disassembled.

\section{Definitions and Representation}

\subsection{Definitions}

We shall now define a set of concepts:

- Components: These are elements with an individual physical entity which form a product, e.g. brakes, tyres.

- Contact elements: These are elements with a physical independent entity which link two or more components, e.g. screws, cables.

- Unions: Composition of several components. There are two types:

1. "Closure unions": These permit a composition among components and/or contact elements which together form an assembly.

2. "Contact unions": These exist when closure unions at a high hierarchical level exist.

- Sub-assembly: A group of components or contact elements joined together as a whole which can be considered as a new component with a high granularity level.

- Final assembly: This is the union of all the components, sub-assemblies and contact elements that form a product.

- Product: This is the final assembly.

\subsection{Representation}

The previous elements are represented graphically in the following way:

- Graph: Graphical representation of nodes and edges of an assembly or a product.

- Node: Graphical representation of each component (with a circle) and contact elements (with a double circle) which form the product.

- Edge: Graphical representation of the unions. Closure unions are indicated by a continuous line; contact unions are indicated by a dotted line.

- Assembly: This is represented by a group of nodes and/or edges that can be joined by at least one closure union. Taking the hierarchical relations into consideration, a node that is part of a sub-assembly $i$ can be part of another subassembly $j$ if both sub-assemblies $i$ and $j$ can change their order (Fig. 1(a)). If they cannot change their order - in other words, if there is a precedence of the sub-assembly $i$ (a)

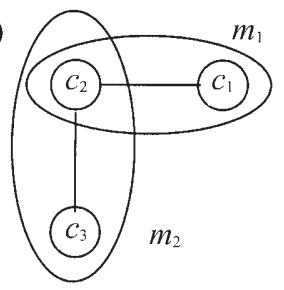

First $m_{1}$ and then $m_{2}$ or vice-versa, it doesn't matter.

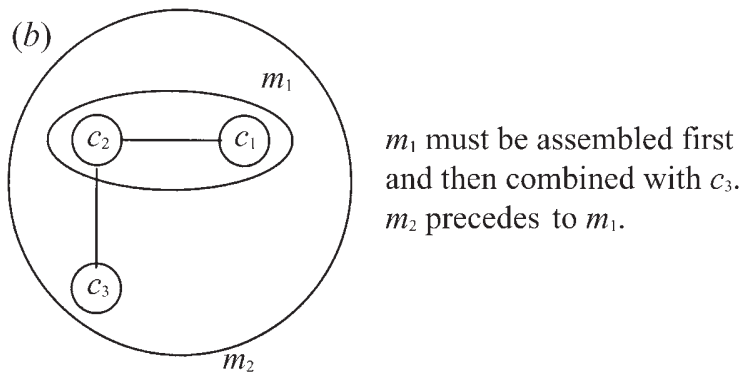

Fig. 1. Relations between sub-assemblies. (a) Non-precedence subassemblies. (b) Precedence sub-assemblies ( $m_{2}$ precedes $\left.m_{1}\right)$.

against the sub-assembly $j$ - the common node will be part of the sub-assembly $j$, which is of a lower precedence. The rest of the components of the sub-assembly $i$ are combined with the sub-assembly $j$, which is considered as an individual component, so that all the components stay within the subassembly $i$ (Fig. 1(b)).

\section{Disassembly Methodology}

A product is made up of a group of interconnected components and/or sub-assemblies. A sub-assembly is also composed of a group of interconnected components and/or sub-assemblies. In both cases the representations are made up using a graph. Taking the previous definitions into consideration, there are different types of relations among the components and the subassemblies. Each of these situations corresponds to a specific disassembly operation, which we shall call an action.

\subsection{Disassembly Actions}

The disassembly actions are now described:

1. Closure union among components and/or sub-assemblies; precedence among sub-assemblies (Fig. 2(a)). The action to be carried out will remove the union and separate its components.

2. Contact union among components:

(a) Between two components (Fig. 2(b)). The action consists of separating the two components from each other.

(b) Among more than two components (Fig. 2(c)). The action consists of separating the components from one of the ends, until we arrive at the desired node. 
(a)

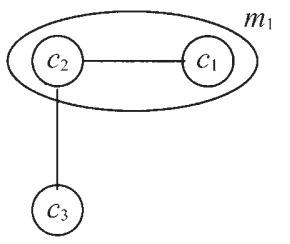

Representation

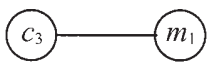

(b)

Representation

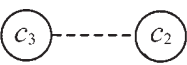

(c)

Representation

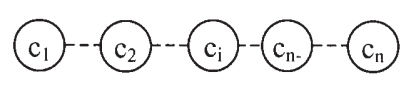

(d)

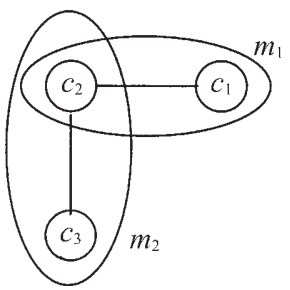

Representation

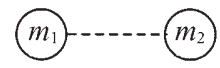

(e)

Representation

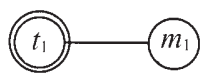

(f)

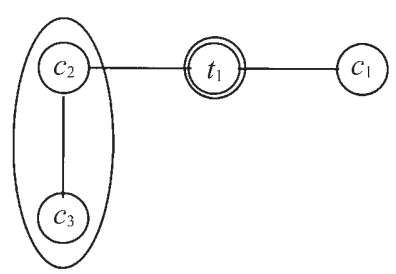

Representation

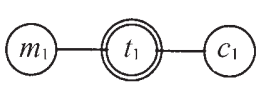

(g)

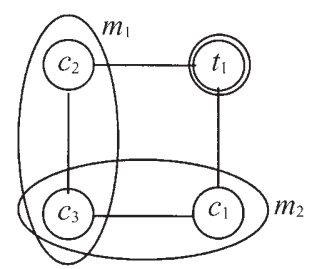

\section{Action}

Separate $c_{1}$ and $c_{2}$, and follow until $c_{i}$. Or $c_{n}$ and $c_{n-1}$ to $c_{i}$

\section{Action}

Nothing

\section{Action}

Remove $t_{1}$ $\underline{\text { Action }}$

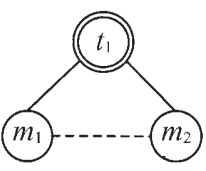

$t_{1}$
Action

Remove the fastener $t_{1}$ and then separate $c_{1}$ and $m_{1}$

Remove the fastener

Fig. 2. Representation and disassembly actions of the different types of sub-assemblies.

3. Assemblies without precedence among them (Fig. 2(d)). In this case there is nothing to be done.

4. Closure union between sub-assembly and contact element (Fig. 2(e)). In this case the contact element has to be removed.

5. Sub-assembly and/or component unions through a contact element (Fig. 2(f)). In this case the contact element has to be removed and then the subassemblies/components which where united by the contact element are separated.

6. Sub-assemblies united by an external contact element whose disassembly sequence is interchangeable (Fig. $2(g)$ ). In this case, the action to be done consists of removing the contact element. 


\subsection{Disassembly Sequence}

When the graph of a product is made, using the definitions and the rules cited previously, it is possible to compute the correct partial disassembly sequence of the product by following the steps described in the following paragraphs.

The algorithm, which sets the disassembly sequence of a component $c_{j}$ or of a sub-assembly $m_{i}$ of the product, has the following steps:

- Step 1. If a component $c_{j}$ is to be disassembled, in this step of the algorithm nothing is to be done. When a sub-assembly $m_{i}$ is to be disassembled, however, it is considered as a new component. Nevertheless, a component can be part of several sub-assemblies. If the $n$ components that are part of the subassembly $m_{i}$ and they are also part of other subassemblies then the graph will have to be restructured. The entire sub-assembly $m_{i}$ is considered as a new component and has to form part of the other sub-assemblies to which its components belong. For example, if the disassembly $m_{1}$ in the graph shown in Fig. 3(a), has to be disassembled, the graph has to be restructured so that it is finally expressed as shown in Fig. 3(b). Therefore, for each of the $n$ components of the sub-assembly $m_{i}$ it is necessary to check if it also forms part of the other $m-1$ sub-assemblies in the graph. If so, the operation previously described is carried out. The cost of this operation is $O(n * m)$, where $n$ is the number of components in the sub-assembly $m_{i}$, and $m$ is the number of sub-assemblies in the graph.

- Step 2. Identify the sub-assemblies to which the component $c_{j}$ to be disassembled belongs and then identify the action to be done to remove the component $c_{j}$. Therefore, it is necessary to find to which of the $m$ sub-assemblies in the graph the component $c_{j}$ belongs, to obtain a group $S$ of sub-assemblies to which the component $c_{j}$ belongs. Identify the actions associated with the removal of the component $c_{j}$ of each of the sub-assemblies of the group $S$. As the component $c_{j}$ is in all of the subassemblies at the same hierarchy level, the disassembly actions obtained can be done in parallel or the order of execution among them can be interchanged. This step has a cost of $O(m)$.

- Step 3. Carry out step 2, considering all the sub-assemblies of the group $S$ as a new component to be disassembled. On carrying out this step, a new group $S^{\prime}$ will have been generated, which is composed of sub-assemblies to which the sub-assemblies of the group $S$ belong. Therefore, it is necessary to repeat step 2 until a group composed only of the final assembly is obtained.

- Step 4. This process has set a path to be followed in the reverse order, removing the closure unions found until we have arrived at the source node.

This method gives a disassembly sequence to be followed for disassembling a component of the product, or to disassemble a sub-assembly of the product.

\section{Results}

A program has been implemented which permits the introduction of product graphs that indicate the components belonging to each sub-assembly. The output of the program is the partial disassembly sequence of the product.

As an example of its application, the disassembly of a remote control with five components and one contact element (a screw which joins four of the components) is presented. After this example the disassembly of a full PC with all its components is presented.

\subsection{Disassembly Sequence of a Remote Control}

In Fig. 4(a) we show a synthetic image of the remote control. It is made up of five components and one contact element. Component $c_{4}$ is the rubber keyboard, $c_{3}$ is the integrated circuit which has the electronics of the remote control, components $c_{2}$ and $c_{5}$ have the two previous components sandwiched between them and a screw at the top of $c_{2}$ which keeps the four components together. $c_{1}$, which is the cover plate of the batteries, is located on top of $c_{2}$. The unions between $c_{2}-c_{3}$, $c_{3}-c_{4}$ and $c_{4}-c_{5}$ are contact unions, and exist only while the closure union between $c_{2}-c_{5}$ exists.

In Fig. 4(b) we show the product graph labelled with the different sub-assemblies. The sub-assembly $m_{1}$ is made up by
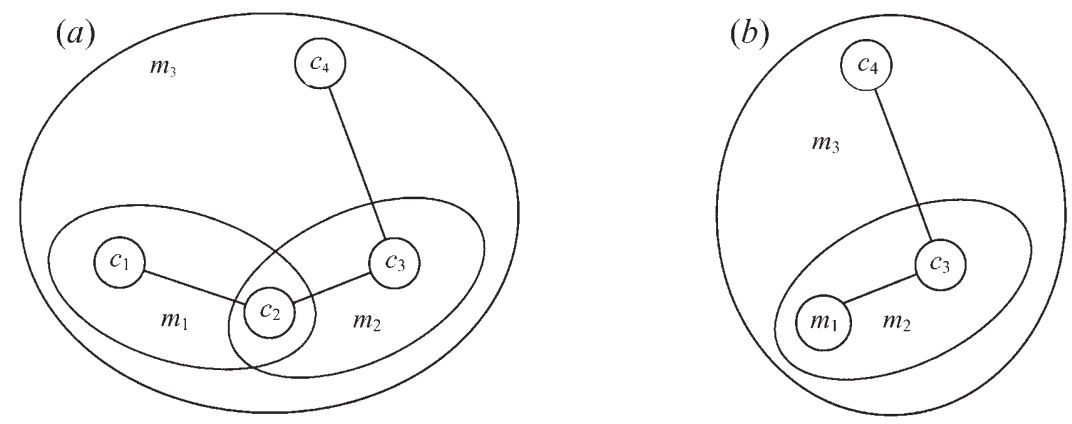

Fig. 3. Sub-assembly reconstruction. 

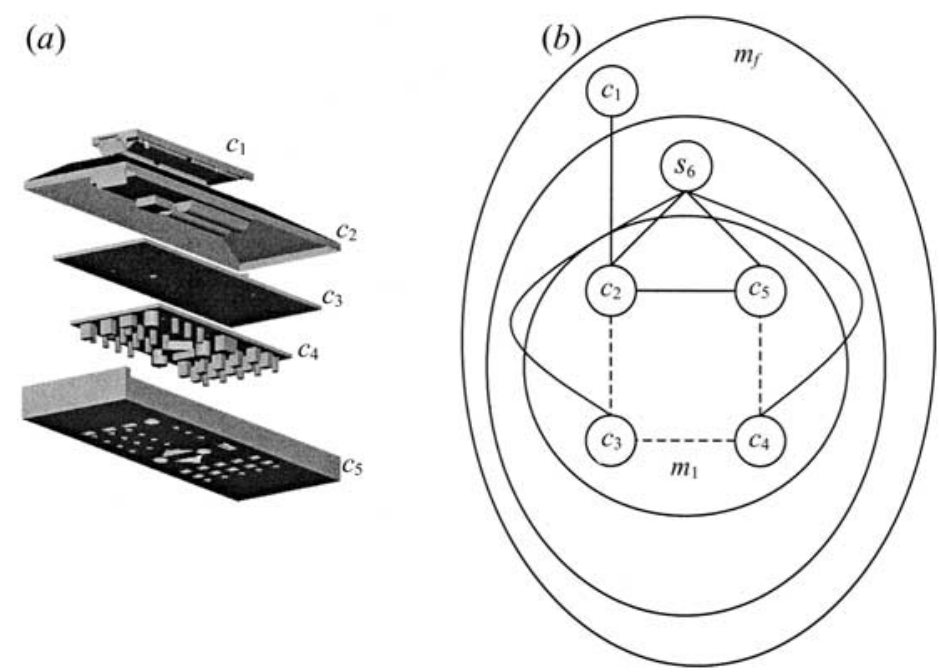

Fig. 4. Remote-control scheme and its graph.

the union of $c_{2}, c_{3}, c_{4}$ and $c_{5}$ which are joined by a closure union between $c_{2}$ and $c_{5}$. The sub-assembly $m_{2}$ is composed of the sub-assembly $m_{1}$ and the screw which joins the components of this sub-assembly $\left(s_{6}\right)$. The final assembly of the product is composed of the sub-assembly $m_{2}$ and the component $c_{1}$.

In Fig. 5(a) we present the product graph introduced in the program. In Fig. 5(b) each of the sub-assemblies of the product are indicated as well as the actions related to each one, according to the rules previously established.

When the product is represented, the disassembly sequence has to be computed using the algorithm in Section 4.2.

First the disassembly sequence for the component $3\left(c_{3}\right)$ is computed, taking the action list in Fig. 5(b) into consideration. The actions to be done are the following:

- Step 1 is not used because we are trying to disassemble just one component, not a sub-assembly. The result after iterating the steps 2 and 3 of the algorithm are:

$-c_{3} \in m_{1}=>$ The action to be done can be expressed as follows: Remove union and separate $c_{2}$ and $c_{5}$ and use action 2b: separate $c_{2}$ and $c_{3}$ or separate $c_{5}$ and $c_{4}$ and then $c_{4}$ and $c_{3}$.

- $m_{1} \in m_{2}=>$ Remove $s_{6}$.

$-m_{2} \in m_{\mathrm{f}}=>$ Remove union and separate $c_{1}$ and $m_{2}$.

- Finally, step 4 of the algorithm establishes the actions to be done for disassembling $c_{3}$ :

- Remove union and separate $c_{1}$ and $m_{2}$.

- Remove $s_{6}$.

- Remove union and separate $c_{2}$ and $c_{5}$.

- Separate $c_{2}$ and $c_{3}$.

- Disassemble $c_{3}$.

In the same way, the actions for disassembling component 2 $\left(c_{2}\right)$ are the following:

- Step 1: There is no need to use it because what is to be disassembled is a component and not a sub-assembly.

- Steps 2 and 3:

$-c_{2} \in m_{1}=>$ Remove union and separate $c_{2}$ and $c_{5}$.
- $m_{1} \in m_{2}=>$ Remove $s_{6}$.

- $m_{2} \in m_{\mathrm{f}}=>$ Remove union and separate $c_{1}$ and $m_{2}$.

- Step 4:

- Remove union and separate $c_{1}$ and $m_{2}$.

- Remove the screw $s_{6}$.

- Remove union and separate $c_{2}$ and $c_{5}$.

- Disassemble $c_{2}$.

\subsection{Disassembly Sequence of a PC}

In Fig. 6(a) the graph of a full PC is presented. It is composed of several components, detailed in Table 1. The different assemblies and the components or sub-assemblies that belong to each assembly are presented in Table 2 .

For disassembling the hard disk $\left(c_{32}\right)$ we have to disconnect it from all the components that are connected to it. In this way, the disassembly tree shown in Fig. 6(b) is obtained. As shown in this tree, the tasks to be done for disassembling the hard disk are the following, according to the algorithm:

- Step 1: There is no need to use it because what is to be disassembled is a component and not an sub-assembly.

- Steps 2 and 3:

$-c_{32} \in m_{50}=>$ Remove $c_{35}$.

$-c_{32} \in m_{54}=>$ Remove union and separate $c_{32}$ and $c_{29}$.

$-c_{32} \in m_{18}=>$ Remove $c_{52}$ and separate $c_{1}$ and $c_{32}$.

$-m_{18}, m_{50}, m_{54} \in m_{45}=>$ Nothing.

$-m_{45} \in m_{46}=>$ Remove $c_{13}$ and separate $m_{45}$ and $c_{14}$.

- Step 4:

- Remove $c_{13}$.

- Separate $m_{45}$ and $c_{14}$.

- The following operations can be done in parallel:

- Remove $c_{52}$. Then separate $c_{1}$ and $c_{32}$.

- Remove $c_{35}$.

- Remove union and separate $c_{32}$ and $c_{29}$.

- Disassemble $c_{32}$

In Fig. 6(b) the tasks that can be done in parallel, with a reduction in the disassembly cost of the product, are shown. 
(a)

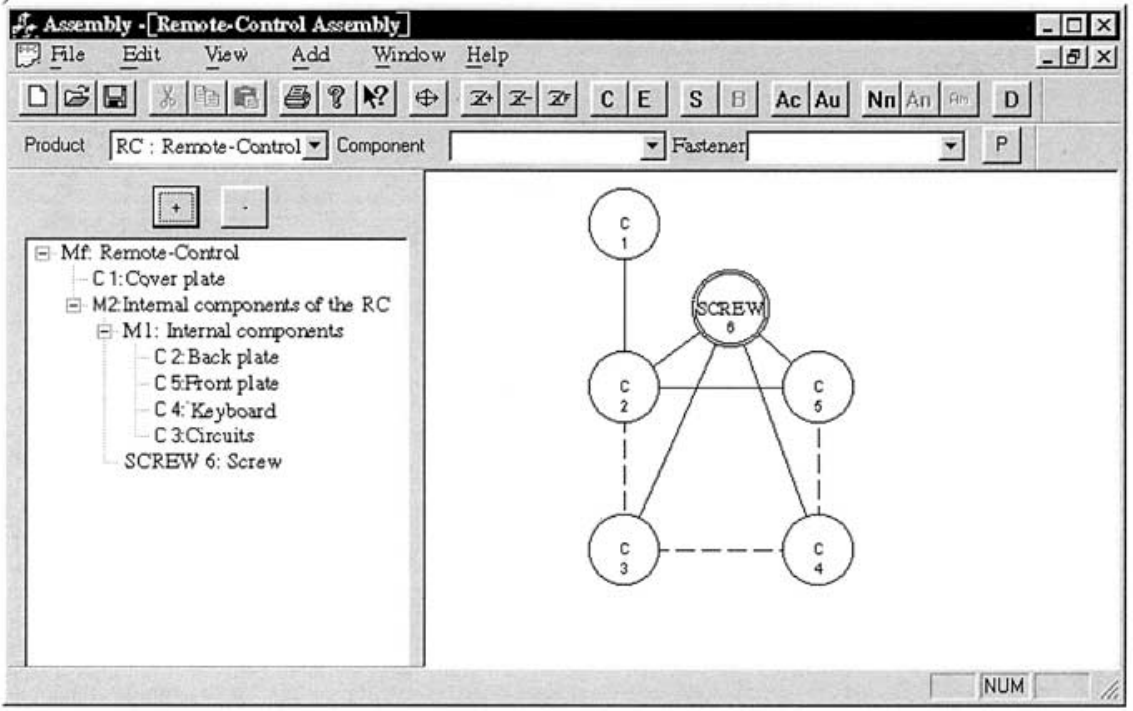

(b)

\begin{tabular}{|c|c|c|}
\hline$m_{f}=\left\{c_{1}, m_{2}\right\}$ & $\underline{\text { Representation }}$ & $\begin{array}{l}\frac{\text { Action }}{\text { (1) Remove union and }} \\
\text { separate } c_{1} \text { and } m_{2}\end{array}$ \\
\hline$m_{2}=\left\{s_{6}, m_{1}\right\}$ & & (4) Remove $s_{6}$ \\
\hline$m_{1}=\left\{c_{2}, c_{3}, c_{4}, c_{5}\right\}$ & & $\begin{array}{l}\text { (1) Remove union and } \\
\text { separate } c_{2} \text { and } c_{5} \\
\text { (2b) Apply } \\
\text { according to the target }\end{array}$ \\
\hline
\end{tabular}

Fig. 5. Remote control. (a) Draw in the program. (b) Actions of each sub-assembly.

The tasks to be done for disassembling the mother board $\left(c_{2}\right)$ together with the cache $\left(c_{28}\right)$, which implies the disassembly of the sub-assembly $m_{12}$, are the following:

- Step 1: Reduce the graph replacing the appearances of the component $c_{2}$ and the component $c_{28}$ with the sub-assembly $m_{12}$ and simplify the graph (its relations), considering the sub-assembly $m_{12}$ as a component.

- Steps 2 and 3:

- $m_{12} \in m_{0}=>$ Remove union and separate $c_{7}$ and $m_{12}$.

- $m_{12} \in m_{1}=>$ Remove union and separate $c_{6}$ and $m_{12}$.

$-m_{12} \in m_{2}=>$ Remove union and separate $c_{5}$ and $m_{12}$.

- $m_{12} \in m_{3}=>$ Remove union and separate $c_{4}$ and $m_{12}$.

- $m_{12} \in m_{4}=>$ Remove union and separate $c_{3}$ and $m_{12}$.

- $m_{12} \in m_{8}=>$ Remove union and separate $c_{24}$ and $m_{12}$.

- $m_{12} \in m_{9}=>$ Remove union and separate $c_{25}$ and $m_{12}$.

- $m_{12} \in m_{10}=>$ Remove union and separate $c_{26}$ and $m_{12}$.

$-m_{12} \in m_{11}=>$ Remove union and separate $c_{27}$ and $m_{12}$.

- $m_{12} \in m_{28}=>$ Remove union and separate $m_{12}$ and $c_{46}$. $-m_{12} \in m_{29}=>$ Remove $c_{49}$ and separate $c_{48}$ and $m_{12}$.

$-m_{12} \in m_{41}=>$ Remove $c_{51}$ and separate $m_{12}$ and $c_{1}$.

$-m_{12} \in m_{47}=>$ Remove $c_{35}$.

$-m_{12} \in m_{48}=>$ Remove $c_{36}$.

- $m_{12} \in m_{57}=>$ Remove union and separate $c_{29}$ and $m_{12}$.

$-m_{12} \in m_{58}=>$ Remove union and separate $c_{15}$ and $m_{12}$.

- $m_{12} \in m_{59}=>$ Remove union and separate $c_{16}$ and $m_{12}$.

- $m_{12} \in m_{60}=>$ Remove union and separate $c_{17}$ and $m_{12}$.

- $m_{29} \in m_{30}=>$ Remove $c_{50}$ and separate $m_{29}$ and $c_{1}$.

- $m_{0}, m_{30} \in m_{31}=>$ Remove $c_{8}$.

- $m_{1}, m_{30} \in m_{32}=>$ Remove $c_{9}$.

- $m_{2}, m_{30} \in m_{33}=>$ Remove $c_{10}$.

- $m_{3}, m_{30} \in m_{34}=>$ Remove $c_{11}$.

- $m_{4}, m_{30} \in m_{35}=>$ Remove $c_{12}$.

- $m_{8}, m_{9}, m_{10}, m_{11}, m_{12}, m_{28}, m_{31}, m_{32}, m_{33}, m_{34}, m_{35}, m_{41}$, $m_{47}, m_{48}, m_{57}, m_{58}, m_{59}, m_{60} \in m_{45}=>$ Nothing.

- $m_{45} \in m_{46}=>$ Remove $c_{13}$ and separate $m_{45}$ and $c_{14}$.

- Step 4: In this sequence there are several decisions to be made depending on the parallelism of the system: 


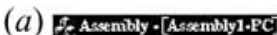

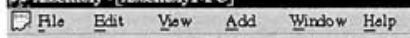

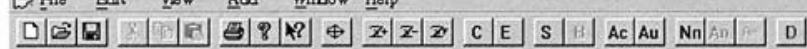

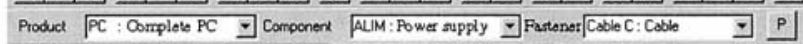

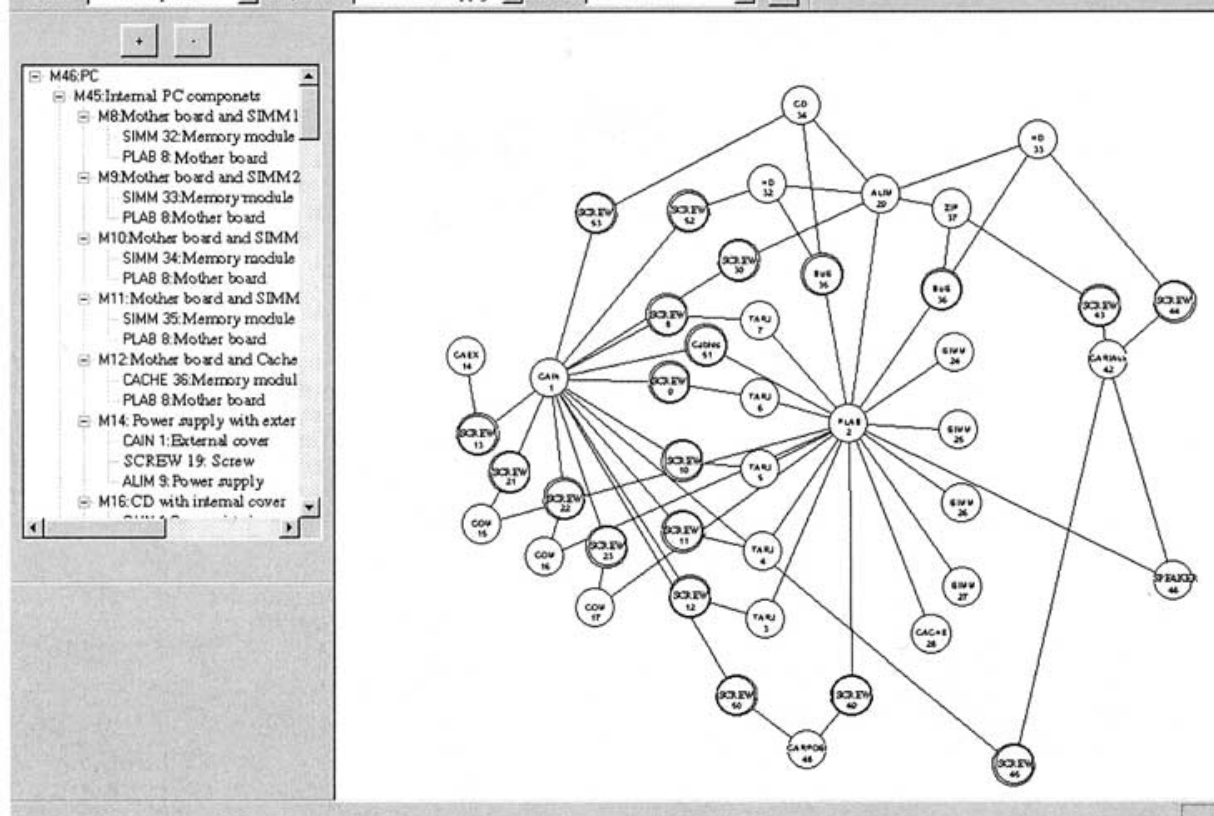

(b)

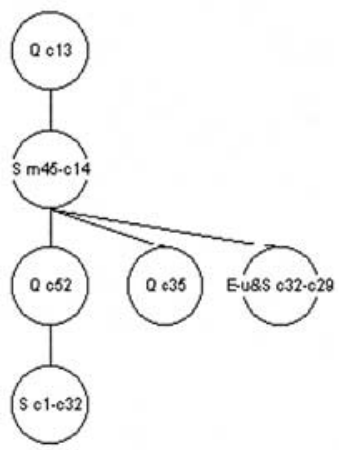

(c)

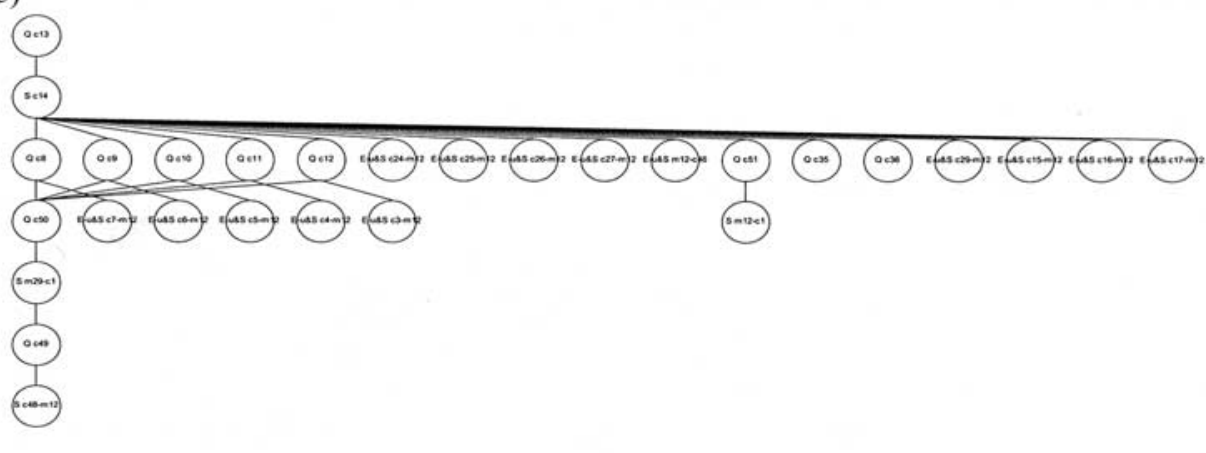

Fig. 6. PC. (a) Scheme of the PC in the program. (b) Disassembly tree of the hard disk. (c) Disassembly tree of the sub-assembly $m_{12}$.

- Remove $c_{13}$.

- Separate $m_{45}$ and $c_{14}$.

The following operations can be done in parallel:

- Remove $c_{8}$.

- Remove $c_{9}$.

- Remove $c_{10}$.

- Remove $c_{11}$.

- Remove $c_{12}$.

- Remove $c_{50}$.

- Separate $m_{29}$ and $c_{1}$.

- The following operations can be done in parallel:

- Remove union and separate $c_{7}$ and $m_{12}$.

- Remove union and separate $c_{6}$ and $m_{12}$.

- Remove union and separate $c_{5}$ and $m_{12}$.

- Remove union and separate $c_{4}$ and $m_{12}$.

- Remove union and separate $c_{3}$ and $m_{12}$.
- Remove union and separate $c_{24}$ and $m_{12}$.

- Remove union and separate $c_{25}$ and $m_{12}$.

- Remove union and separate $c_{26}$ and $m_{12}$.

- Remove union and separate $c_{27}$ and $m_{12}$.

- Remove union and separate $m_{12}$ and $c_{46}$.

- Remove $c_{49}$. Then separate $c_{48}$ and $m_{12}$.

- Remove $c_{51}$. Then separate $m_{12}$ and $c_{1}$.

- Remove $c_{35}$.

- Remove $c_{36}$.

- Remove union and separate $c_{29}$ and $m_{12}$.

- Remove union and separate $c_{15}$ and $m_{12}$.

- Remove union and separate $c_{16}$ and $m_{12}$.

- Remove union and separate $c_{17}$ and $m_{12}$.

- Disassemble $m_{12}$.

In Fig. 6(c), the disassembly sequence tree is shown. It is the disassembly solution for the disassembly of the motherboard 
Table 1. List of components of the PC.

\begin{tabular}{|c|c|c|}
\hline Component & Description & Union \\
\hline 1 & Interior box & No \\
\hline 2 & Motherboard & No \\
\hline 3 & TV card & No \\
\hline 4 & Net card & No \\
\hline 5 & SCSI card & No \\
\hline 6 & Sound card & No \\
\hline 7 & Video card & No \\
\hline 8 & Screw & Yes \\
\hline 9 & Screw & Yes \\
\hline 10 & Screw & Yes \\
\hline 11 & Screw & Yes \\
\hline 12 & Screw & Yes \\
\hline 13 & Screw & Yes \\
\hline 14 & Exterior box & No \\
\hline 15 & Communications port COM1 & No \\
\hline 16 & Communications port COM2 & No \\
\hline 17 & Communications port LPT1 & No \\
\hline 21 & Screw & Yes \\
\hline 22 & Screw & Yes \\
\hline 23 & Screw & Yes \\
\hline 24 & Memory module, SIMM 1 & No \\
\hline 25 & Memory module, SIMM 2 & No \\
\hline 26 & Memory module, SIMM 3 & No \\
\hline 27 & Memory module, SIMM 4 & No \\
\hline 28 & Memory module, cache & No \\
\hline 29 & Power supply & No \\
\hline 30 & Screw & Yes \\
\hline 32 & Hard disk (HD) & No \\
\hline 33 & Hard disk (HD) & No \\
\hline 34 & Compack disk (CD) & No \\
\hline 35 & Data bus IDE 1 & Yes \\
\hline 36 & Data bus IDE 2 & Yes \\
\hline 37 & Remocable disk unit, ZIP & No \\
\hline 42 & Auxiliary box from leaving disk units & No \\
\hline 43 & Screw & Yes \\
\hline 44 & Screw & Yes \\
\hline 45 & Screw & Yes \\
\hline 46 & PC Internal speaker & No \\
\hline 48 & Rear box & No \\
\hline 49 & Screw & Yes \\
\hline 50 & Screw & Yes \\
\hline 51 & Generic cables & Yes \\
\hline 52 & Screw & Yes \\
\hline 53 & Screw & Yes \\
\hline
\end{tabular}

together with the cache memory, in other words, the subassembly $m_{12}$.

\section{Conclusions}

The algorithm presented can compute a disassembly sequence of a product automatically, based only on the information introduced by an operator who knows the product. This information can be obtained from the design process.

This method can be used in new product design as well as for the recycling or maintenance of existing products that are not designed for disassembly.

It achieves a disassembly schedule which permits a nondestructive disassembly of the products for their later maintenance or recycling.
Table 2. List of sub-assemblies of the PC.

\begin{tabular}{|c|c|c|}
\hline Sub-assembly & Description & $\begin{array}{l}\text { Component/ } \\
\text { Sub-assembly }\end{array}$ \\
\hline 0 & Motherboard with video card & $\begin{array}{l}7 \\
2\end{array}$ \\
\hline 1 & Motherboard with sound card & $\begin{array}{l}6 \\
2\end{array}$ \\
\hline 2 & Motherboard with SCSI card & $\begin{array}{l}5 \\
2\end{array}$ \\
\hline 3 & Motherboard with net card & $\begin{array}{l}4 \\
2\end{array}$ \\
\hline 4 & Motherboard with TV card & $\begin{array}{l}3 \\
2\end{array}$ \\
\hline 8 & Motherboard with SIMM 1 & $\begin{array}{l}24 \\
2\end{array}$ \\
\hline 9 & Motherboard with SIMM 2 & $\begin{array}{l}25 \\
2\end{array}$ \\
\hline 10 & Motherboard with SIMM 3 & $\begin{array}{l}26 \\
2\end{array}$ \\
\hline 11 & Motherboard with SIMM 4 & $\begin{array}{l}27 \\
2\end{array}$ \\
\hline 12 & Motherboard with Cache & $\begin{array}{l}28 \\
2\end{array}$ \\
\hline 14 & Power supply with interior box & $\begin{array}{l}1 \\
30 \\
29\end{array}$ \\
\hline 16 & $\mathrm{CD}$ with interior box & $\begin{array}{l}1 \\
53 \\
34\end{array}$ \\
\hline 18 & HD1 with box & $\begin{array}{l}1 \\
52 \\
32\end{array}$ \\
\hline 23 & HD2 with box & $\begin{array}{l}42 \\
44 \\
33\end{array}$ \\
\hline 26 & ZIP with box & $\begin{array}{l}42 \\
43 \\
37\end{array}$ \\
\hline 27 & Speaker with box & $\begin{array}{l}46 \\
42\end{array}$ \\
\hline 28 & Speaker with motherboard & $\begin{array}{l}2 \\
46\end{array}$ \\
\hline 29 & Motherboard with rear box & $\begin{array}{l}48 \\
49 \\
2\end{array}$ \\
\hline 30 & Interior box with motherboard & $\begin{array}{l}50 \\
1 \\
\text { M29 }\end{array}$ \\
\hline 31 & Interior box with video card & $\begin{array}{l}8 \\
\text { M0 } \\
\text { M30 }\end{array}$ \\
\hline 32 & Interior box with sound card & $\begin{array}{l}9 \\
\text { M1 } \\
\text { M30 }\end{array}$ \\
\hline 33 & Interior box with SCSI card & $\begin{array}{l}10 \\
\mathrm{M} 2 \\
\mathrm{M} 30\end{array}$ \\
\hline 34 & Interior box with net card & $\begin{array}{l}11 \\
\text { M3 } \\
\text { M30 }\end{array}$ \\
\hline 35 & Interior box with TV card & $\begin{array}{l}12 \\
\text { M4 } \\
\text { M30 }\end{array}$ \\
\hline 36 & Interior box with COM1 & $\begin{array}{l}15 \\
21 \\
1\end{array}$ \\
\hline 37 & Interior box with $\mathrm{COM} 2$ & $\begin{array}{l}1 \\
22 \\
16\end{array}$ \\
\hline
\end{tabular}


Table 2. Continued

\begin{tabular}{|c|c|c|}
\hline Sub-assembly & Description & $\begin{array}{l}\text { Component/ } \\
\text { Sub-assembly }\end{array}$ \\
\hline 38 & Interior box with LPT1 & $\begin{array}{l}1 \\
23 \\
17\end{array}$ \\
\hline 41 & $\begin{array}{l}\text { Interior box with motherboard } \\
\text { through cables }\end{array}$ & $\begin{array}{l}2 \\
51 \\
1\end{array}$ \\
\hline 42 & Interior box with auxiliary box & $\begin{array}{l}42 \\
45 \\
1\end{array}$ \\
\hline 44 & Auxiliary box with its components & $\begin{array}{l}\text { M23 } \\
\text { M26 } \\
\text { M27 } \\
\text { M42 }\end{array}$ \\
\hline 45 & Internal components of the $\mathrm{PC}$ & $\begin{array}{l}\text { M8 } \\
\text { M9 } \\
\text { M10 } \\
\text { M11 } \\
\text { M12 } \\
\text { M14 } \\
\text { M16 } \\
\text { M18 } \\
\text { M28 } \\
\text { M31 } \\
\text { M32 } \\
\text { M33 } \\
\text { M34 } \\
\text { M35 } \\
\text { M36 } \\
\text { M37 } \\
\text { M38 } \\
\text { M41 } \\
\text { M44 } \\
\text { M47 } \\
\text { M48 } \\
\text { M49 } \\
\text { M50 } \\
\text { M51 } \\
\text { M52 } \\
\text { M53 } \\
\text { M54 } \\
\text { M55 } \\
\text { M56 } \\
\text { M57 } \\
\text { M58 } \\
\text { M59 } \\
\text { M60 }\end{array}$ \\
\hline 46 & Full PC & $\begin{array}{l}13 \\
14 \\
\text { M45 }\end{array}$ \\
\hline 47 & Motherboard with data bus & $\begin{array}{l}35 \\
2\end{array}$ \\
\hline 48 & Motherboard with data bus & $\begin{array}{l}36 \\
2\end{array}$ \\
\hline 49 & CD with data bus & $\begin{array}{l}35 \\
34\end{array}$ \\
\hline 50 & HD 1 with data bus & $\begin{array}{l}35 \\
32\end{array}$ \\
\hline 51 & HD 2 with data bus & $\begin{array}{l}36 \\
33\end{array}$ \\
\hline 52 & ZIP with data bus & $\begin{array}{l}37 \\
36\end{array}$ \\
\hline 53 & CD with power supply & $\begin{array}{l}34 \\
29\end{array}$ \\
\hline 54 & HD 1 with power supply & $\begin{array}{l}32 \\
29\end{array}$ \\
\hline
\end{tabular}

(Continued)
Table 2. Continued

$\begin{array}{lll}\text { Sub-assembly } & \text { Description } & \begin{array}{l}\text { Component/ } \\ \text { Sub-assembly }\end{array} \\ & & 33 \\ 55 & \text { HD 2 with power supply } & 29 \\ 56 & \text { ZIP with power supply } & 37 \\ & & 29 \\ 57 & \text { Motherboard with power supply } & 29 \\ & & 2 \\ 58 & \text { Motherboard with COM 1 bus } & 15 \\ & & 2 \\ 59 & \text { Motherboard with COM 2 bus } & 16 \\ & & 2 \\ 60 & \text { Motherboard with LPT 1 bus } & 17 \\ & & 2\end{array}$

This algorithm is being implemented in an automatic and autonomous disassembly system to obtain the schedule and the subsequent disassembly of the products.

\section{Acknowledgement}

This work was funded by the Spanish CICYT project "Sistema Robotizado de Desensamblado Automático basado en Modelos y Visión Artificial" (TAP1999-0436).

\section{References}

1. B. O'Shea, S. S. Grewal and H. Kaebernick, "State of the art literature survey on disassembly planning", Concurrent Engineering: Research and Applications, 6(4), pp. 345-357, 1998.

2. A. K. Subramani and P. Dewhurst, "Automatic generation of product disassembly sequences", Annals of the CIRP, 40(1), pp. 115-118, 1991.

3. K. Yokota and D. R. Brough, "Assembly/disassembly sequence planning", Assembly Automation, 12(3), pp. 31-38, 1992.

4. W. H. Qian and E. Pagello, "On the scenario and heuristics of disassemblies", 1994 IEEE International Conference on Robotics and Automation, pp. 264-271, 1994.

5. H. Srinivasan and R. Gadh, "Selective disassembly of components with geometric constraints", Proceedings of the ASME Design Engineering Technical Conference, Las Vegas, NV, 1999.

6. N. Shyamsundar and R. Gadh, "Geometric abstractions to support disassembly analysis". IEE Transactions, 31, pp. 935-946, 1999.

7. K. Lee and R. Gadh, "Destructive disassembly to support virtual prototyping”, IEE Transactions, 30, pp. 959-970, 1998.

8. H. Srinivasan and R. Gadh, "A geometric algorithm for single selective disassembly using the wave propagation abstraction", Computer-Aided Design, 30(8), pp. 603-613, 1998.

9. H. Srinivasan and R. Gadh, "Complexity reduction in geometric selective disassembly using the wave propagation abstraction", International Conference on Robotics and Automation (ICRA), Leuven, Belgium, 1998.

10. H. Srinivasan, R. Figueroa and R. Gadh, "Selective disassembly for virtual prototyping as applied to de-manufacturing", Robotics and Computer Integrated Manufacturing, 15, pp. 231-245, 1999.

11. M. Martinez, V.-H. Pham and J. Favrel, "Dynamic generation of disassembly sequences", IEEE International Conference on Emerging Technologies and Factory Automation, ETFA'97, pp. 177$182,1997$.

12. H. Perlewitz, K. Müller and G. Seliger, "Usage mode and effects analysis to support disassembly process planning", IFAC Intelligent Assembly and Dissasembly, Bled, Slovenia, pp. 11-16, 1998. 
13. S. Lee and C. Yi, "Forced-based reasoning in assembly planning", Vision Sensors and Control for Autmated Manufacturing Systems, SPIE, 2063, 97-108 1994.

14. L. S. Homem de Mello and A. C. Sanderson, "AND/OR graph representation of assembly plans", IEEE Transactions on Robotics and Automation, 6(2), pp. 188-199, April 1990.

15. S. Zorc, "Learning in assembly planning", IFAC Intelligent Assembly and Dissasembly, Bled, Slovenia, pp. 17-22, 1998.

16. E. Zussman, E. Lenz and M. Shpitalni, "An approach to the automatic assembly planning problem", Annals of the CIRP, 39(1), pp. 33-36, 1990

17. H. C. Zhang and T. C. Kuo, "A graph-based approach to disassembly model for end-of-life product recycling", IEEE/CPMT Electronics Manufacturing Technology Symposium, pp. 274-254, 1996.
18. H. C. Zhang and T. C. Kuo, "A graph-based disassembly sequence planning for EOL product recycling", IEEE/CPMT Electronics Manufacturing Technology Symposium, pp. 140$151,1997$.

19. T. C. Kuo, "Disassembly sequence and cost analysis for electromechanical products", Robotics and Computer Integrated Manufacturing, 16, pp. 43-54, 2000.

20. B. O'Seha, H. Kaebernick and S. S. Grewal, "Using a cluster graph representation of products for application in the disassembly planning process", Concurrent Engineering: Research and Applications, 8(3), pp. 158-170, 2000.

21. S. T. Puente, F. Torres and P. Gil, "An approach to disassembly sequence generation", IFAC Intelligent Assembly and Disassembly, Caneda, Brazil, 2001. 